\title{
Guillain-Barré syndrome related to COVID-19 infection
}

Paola Alberti, MD, PhD, * Simone Beretta, MD, PhD,* Marco Piatti, MD, Aristotelis Karantzoulis, MD, Maria Luisa Piatti, MD, Patrizia Santoro, MD, Martina Viganò, MD, Ginevra Giovannelli, MD, Fiammetta Pirro, MD, Danilo Antonio Montisano, MD, Ildebrando Appollonio, MD, and Carlo Ferrarese, MD, PhD

Neurol Neuroimmunol Neuroinflamm 2020;7:e741. doi:10.1212/NXI.0000000000000741

At the time of this writing, healthcare systems are facing worldwide the pandemic of the coronavirus severe acute respiratory coronavirus 2 (SARS-COV-2) and its associated disease, named cronavirus disease 19 (COVID-19). This virus is a new human pathogen, and currently, there are no specific treatment options. ${ }^{1}$ COVID-19 mostly affects the respiratory system, ranging from mild flu-like symptoms to severe pneumonia, but extrarespiratory multisystemic involvement has also been reported. ${ }^{2} \mathrm{Li}$ et al. ${ }^{3}$ recently described the neuroinvasive potential of COVID-19, but, to our knowledge, no case of acute dysimmune neuropathy has been described so far. Here, the authors report the case of an acute and severe peripheral nervous system disorder possibly related to COVID-19 infection.

A 71-year-old male patient was referred to the emergency department for subacute onset of paresthesia at limb extremities, followed by distal weakness rapidly evolving to a severe, flaccid tetraparesis over the previous 3 days. In the previous week, he had low grade fever for a few days. Relevant conditions at his medical history included hypertension, abdominal aortic aneurysm treated with endovascular repair in 2017, and lung cancer treated with surgery only (without additional chemotherapy or radiotherapy) in 2017 with negative oncological follow-up; no previous neurologic history was reported. Neurologic examination showed normal consciousness and language, no cranial nerve deficit, symmetric limb weakness (Medical Research Council score $3 / 5$ at upper limbs and $2 / 5$ at lower limbs), symmetric and extensive stocking-and-glove hypesthesia at the 4 limbs (more pronounced at lower limbs), absent deep tendon reflexes, and normal plantar response. The patients complained of severe paresthesia in both hands and feet. Moderate dyspnea and moderate low back pain were present at the time of the first evaluation. $\mathrm{He}$ showed hemodynamic disturbances with severe drug-resistant hypertension. Arterial blood gases indicated severe hypoxia ( $\mathrm{paO} 265 \mathrm{~mm} \mathrm{Hg}$ without supplemental oxygen). Brain CT scan was normal, whereas chest CT scan showed multiple bilateral ground glass opacities and consolidations, typical of COVID-19 pneumonia. Nasopharyngeal swab tested positive for SARSCoV-2. Lumbar puncture was performed urgently and showed a mild increase in the protein content $(54 \mathrm{mg} / \mathrm{dL})$ and mild leukocytosis $(9$ cells/ $\mu \mathrm{L})$; CSF was negative for SARS-CoV-2. Electroneurography showed the absence of both the sural nerve sensory nerve action potential (SAP) and the tibial nerve compound muscle action potential (CMAP), markedly increased common peroneal CMAP distal latency, markedly decreased velocity, moderately decreased CMAP amplitude (with spatial and temporal dispersion) for the same nerve, and decreased ulnar SAP amplitude. F waves were not performed at lower limbs for the reduced amplitude of the evocable CMAP and not performed at upper limbs for intolerance at the stimulation. See table for a summary of nerve conduction studies results.

\author{
Correspondence \\ Dr. Alberti \\ paola.alberti@unimib.it
}


Table Nerve conduction study parameters

\begin{tabular}{|c|c|c|c|c|}
\hline Nerve & \multicolumn{3}{|c|}{ SAP amplitude (microV) } & Velocity $(\mathrm{m} / \mathrm{sec})$ \\
\hline \multicolumn{5}{|l|}{ Sensory recordings } \\
\hline Sural & & Absent & & Absent \\
\hline Ulnar & & 4.0 & & 52.4 \\
\hline Radial & & 6.4 & & 51.3 \\
\hline Nerve & Distal latency (msec) & CMAP distal amplitude (mV) & CMAP proximal amplitude (mV) & Velocity $(\mathrm{m} / \mathrm{sec})$ \\
\hline \multicolumn{5}{|l|}{ Motor recordings } \\
\hline Tibial & Absent & Absent & Absent & Absent \\
\hline Common peroneal & 16.0 & $1.3^{\mathrm{a}}$ & $0.3^{\mathrm{a}, \mathrm{b}}$ & 23.7 \\
\hline Ulnar & 3.75 & $5.6^{a}$ & $4.3^{\mathrm{a}}$ & 46.3 \\
\hline Radial & 6.05 & $0.4^{\mathrm{a}}$ & $0.1^{a, b}$ & 38.5 \\
\hline
\end{tabular}

Abbreviations: CMAP = compound muscle action potential; SAP = sensory action potential.

Abnormal parameters are highlighted in bold.

a Spatial and temporal CMAP dispersion.

${ }^{\mathrm{b}}$ Conduction block.

Overall, these findings were interpreted as a severe form of acute polyradiculoneuritis with prominent demyelinating features. Diagnosis of Guillain-Barré syndrome (GBS) associated with COVID-19 was made. High-dose IV immunoglobulins $(0.4 \mathrm{~g} / \mathrm{kg} / \mathrm{d}$ for 5 days $)$ were started few hours after admission, together with high-flow $60 \%-80 \%$ oxygen via nonrebreather mask, antiviral therapy (lopinavir + ritonavir), and hydroxychloroquine. Despite these efforts, severe respiratory failure developed during the first 24 hours after admission, unresponsive to continuous positive airway pressure ventilation and prone positioning. The patient died a few hours later because of progressive respiratory failure.

The authors report a possible correlation between acute COVID-19 infection and GBS, a condition that in recent years has been linked to other emergent infections, such as Zika virus. ${ }^{4}$ Our case suggests that COVID-19 may cause peripheral nervous system involvement, even before the resolution of pneumonia, meeting the diagnostic criteria of an acute sensory and motor polyradiculoneuritis. Dysregulation of the immune system due to COVID-19 is not that surprising because McGonagle et al. ${ }^{5}$ recently described systemic hyperinflammation in COVID-19 patients with a macrophage activation syndrome, also known as secondary hemophagocytic lymphohistiocytosis; notably, Quin et al. ${ }^{6}$ also retrospectively evaluated a cohort of 452 patients with COVID-19 observing alterations in lymphocytes.

Early respiratory support, including ICU admission, is indicated but not always feasible during the current pandemic.

The authors agree that a close attention to neurologic complications of COVID-19 is needed, as recently suggested by Mao et al. ${ }^{7}$ The Italian Society of Neurology is currently proposing a multicenter nationwide observational study on neurologic presentations and complications of COVID-19. Similar efforts by other neurologic societies worldwide will benefit both neurologists and patients at a global level.

\section{Acknowledgment}

We thank Giuseppina Resta for her technical assistance for nerve conduction studies.

\section{Study funding}

This study was supported by the Italian Ministry of University and Research grant PRIN 2017CY3J3W.

\section{Disclosure}

The authors have nothing to disclose. Go to Neurology.org/ $\mathrm{NN}$ for full disclosures.

\section{Publication history}

Received by Neurology: Neuroimmunology \& Neuroinflammation April 2, 2020. Accepted in final form April 13, 2020.

Appendix Authors

\begin{tabular}{|c|c|c|}
\hline Name & Location & Contribution \\
\hline $\begin{array}{l}\text { Paola Alberti, } \\
\text { MD, PhD }\end{array}$ & $\begin{array}{l}\text { University of Milano- } \\
\text { Bicocca and Neurology } \\
\text { Dept., San Gerardo } \\
\text { Hospital, ASST MONZA, } \\
\text { Monza, Italy; NeuroMl } \\
\text { (Milan Center for } \\
\text { Neuroscience), Milan, Italy }\end{array}$ & $\begin{array}{l}\text { Data acquisition } \\
\text { Drafting/revising the } \\
\text { manuscript } \\
\text { Analysis or interpretation of } \\
\text { the data }\end{array}$ \\
\hline $\begin{array}{l}\text { Simone } \\
\text { Beretta, MD, } \\
\text { PhD }\end{array}$ & $\begin{array}{l}\text { Neurology Dept., San } \\
\text { Gerardo Hospital, ASST } \\
\text { MONZA and University } \\
\text { of Milano-Bicocca, } \\
\text { Monza, Italy; } \\
\text { NeuroMI (Milan Center } \\
\text { for Neuroscience), } \\
\text { Milan, Italy }\end{array}$ & $\begin{array}{l}\text { Data acquisition } \\
\text { Drafting/revising the } \\
\text { manuscript } \\
\text { Analysis or interpretation of } \\
\text { the dataStudy concept } \\
\text { or design }\end{array}$ \\
\hline
\end{tabular}


Appendix (continued)

\begin{tabular}{|c|c|c|}
\hline Name & Location & Contribution \\
\hline $\begin{array}{l}\text { Marco Piatti, } \\
\text { MD }\end{array}$ & $\begin{array}{l}\text { Neurology Dept., San } \\
\text { Gerardo Hospital, } \\
\text { ASST MONZA, Monza, } \\
\text { Italy }\end{array}$ & Data acquisition \\
\hline $\begin{array}{l}\text { Aristotelis } \\
\text { Karantzoulis, } \\
\text { MD }\end{array}$ & $\begin{array}{l}\text { Neurology Dept., San } \\
\text { Gerardo Hospital, } \\
\text { ASST MONZA, Monza, } \\
\text { Italy }\end{array}$ & $\begin{array}{l}\text { Data acquisition } \\
\text { Analysis or interpretation of } \\
\text { the data }\end{array}$ \\
\hline $\begin{array}{l}\text { Maria Piatti, } \\
\text { MD }\end{array}$ & $\begin{array}{l}\text { Neurology Dept., San } \\
\text { Gerardo Hospital, } \\
\text { ASST MONZA, Monza, } \\
\text { Italy }\end{array}$ & $\begin{array}{l}\text { Data acquisition } \\
\text { Drafting/revising the } \\
\text { manuscript }\end{array}$ \\
\hline $\begin{array}{l}\text { Patrizia M. } \\
\text { Santoro, MD }\end{array}$ & $\begin{array}{l}\text { Neurology Dept., San } \\
\text { Gerardo Hospital, } \\
\text { ASST MONZA, Monza, } \\
\text { Italy }\end{array}$ & $\begin{array}{l}\text { Data acquisition } \\
\text { Drafting/revising the } \\
\text { manuscript }\end{array}$ \\
\hline $\begin{array}{l}\text { Martina } \\
\text { Viganò, MD }\end{array}$ & $\begin{array}{l}\text { Neurology Dept., San } \\
\text { Gerardo Hospital, } \\
\text { ASST MONZA, Monza, } \\
\text { Italy }\end{array}$ & $\begin{array}{l}\text { Data acquisition } \\
\text { Drafting/revising the } \\
\text { manuscript }\end{array}$ \\
\hline $\begin{array}{l}\text { Ginevra } \\
\text { Giovannelli, } \\
\text { MD }\end{array}$ & $\begin{array}{l}\text { Neurology Dept., San } \\
\text { Gerardo Hospital, ASST } \\
\text { MONZA, Monza, Italy }\end{array}$ & $\begin{array}{l}\text { Drafting/revising the } \\
\text { manuscript }\end{array}$ \\
\hline $\begin{array}{l}\text { Fiammetta } \\
\text { Pirro, MD }\end{array}$ & $\begin{array}{l}\text { Neurology Dept., San } \\
\text { Gerardo Hospital, } \\
\text { ASST MONZA, Monza, } \\
\text { Italy }\end{array}$ & Data acquisition \\
\hline $\begin{array}{l}\text { Danilo A. } \\
\text { Montisano, } \\
\text { MD }\end{array}$ & $\begin{array}{l}\text { Neurology Dept., San } \\
\text { Gerardo Hospital, ASST } \\
\text { MONZA, Monza, Italy }\end{array}$ & $\begin{array}{l}\text { Data acquisition } \\
\text { Drafting/revising the } \\
\text { manuscript }\end{array}$ \\
\hline
\end{tabular}

Appendix (continued)

\begin{tabular}{|c|c|c|}
\hline Name & Location & Contribution \\
\hline $\begin{array}{l}\text { Ildebrando } \\
\text { Appollonio, } \\
\text { MD, PhD }\end{array}$ & $\begin{array}{l}\text { University of Milano- } \\
\text { Bicocca and Neurology } \\
\text { Dept., San Gerardo } \\
\text { Hospital, ASST MONZA, } \\
\text { Monza, Italy; NeuroMI } \\
\text { (Milan Center for } \\
\text { Neuroscience), Milan, } \\
\text { Italy }\end{array}$ & $\begin{array}{l}\text { Drafting/revising the } \\
\text { manuscript } \\
\text { Analysis or interpretation of } \\
\text { the data }\end{array}$ \\
\hline $\begin{array}{l}\text { Carlo } \\
\text { Ferrarese, } \\
\text { MD, PhD }\end{array}$ & $\begin{array}{l}\text { Neurology Dept., San } \\
\text { Gerardo Hospital, ASST } \\
\text { MONZA and University } \\
\text { of Milano-Bicocca, } \\
\text { Monza, Italy; NeuroMI } \\
\text { (Milan Center for } \\
\text { Neuroscience), Milan, } \\
\text { Italy }\end{array}$ & $\begin{array}{l}\text { Data acquisition } \\
\text { Drafting/revising the } \\
\text { manuscript } \\
\text { Analysis or interpretation of } \\
\text { the data } \\
\text { Study concept or design } \\
\text { Study Supervision }\end{array}$ \\
\hline
\end{tabular}

\section{References}

1. Casadevall A, Pirofski LA. The convalescent sera option for containing COVID-19. J Clin Invest 2020;130:1545-1548.

2. Wang D, Hu B, Hu C, et al. Clinical characteristics of 138 hospitalized patients with 2019 novel coronavirus-infected pneumonia in Wuhan, China. JAMA 2020;323:1061-1069.

3. $\mathrm{Li}$ YC, Bai WZ, Hashikawa T. The neuroinvasive potential of SARS-CoV2 may play a role in the respiratory failure of COVID-19 patients. J Med Virol 2020;92:552-555.

4. Krauer F, Riesen M, Reveiz L, et al. Zika virus infection as a cause of congenital brain abnormalities and Guillain-barré syndrome: systematic review. PLoS Med 2017;14: e1002203.

5. McGonagle D, Sharif K, O'Regan A, et al. Interleukin-6 use in COVID-19 pneumonia related macrophage activation syndrome. Autoimmun Rev 2020:102537.

6. Qin C, Zhou L, Hu Z, et al. Dysregulation of immune response in patients with COVID-19 in Wuhan, China. Clin Infect Dis Epub 2020 Mar 12.

7. Mao L, Wang M, Chen S et al. Neurological manifestations of hospitalized patients with COVID-19 in Wuhan, China: a retrospective case series study. Available at: doi. org/10.1101/2020.02.22.20026500. Accessed March 28, 2020. 


\title{
Neurology \\ Neuroimmunology \& Neuroinflammation
}

\author{
Guillain-Barré syndrome related to COVID-19 infection \\ Paola Alberti, Simone Beretta, Marco Piatti, et al. \\ Neurol Neuroimmunol Neuroinflamm 2020;7; \\ DOI 10.1212/NXI.0000000000000741
}

This information is current as of April 29, 2020

Neurol Neuroimmunol Neuroinflamm is an official journal of the American Academy of Neurology.

Published since April 2014, it is an open-access, online-only, continuous publication journal. Copyright

Copyright $\odot 2020$ The Author(s). Published by Wolters Kluwer Health, Inc. on behalf of the American Academy of Neurology.. All rights reserved. Online ISSN: 2332-7812.

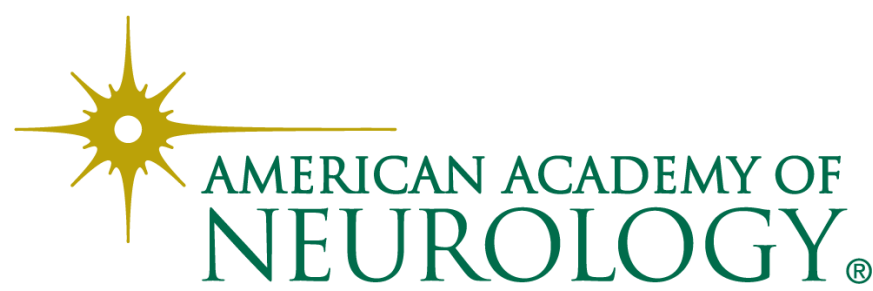




\section{Updated Information \& Services}

References

Citations

Subspecialty Collections

Permissions \& Licensing

Reprints including high resolution figures, can be found at: http://nn.neurology.org/content/7/4/e741.full.html

This article cites 4 articles, 0 of which you can access for free at: http://nn.neurology.org/content/7/4/e741.full.html\#\#ref-list-1

This article has been cited by 20 HighWire-hosted articles: http://nn.neurology.org/content/7/4/e741.full.html\#\#otherarticles

This article, along with others on similar topics, appears in the following collection(s):

Autoimmune diseases

http://nn.neurology.org//cgi/collection/autoimmune_diseases COVID-19

http://nn.neurology.org//cgi/collection/covid_19

Guillain-Barre syndrome

http://nn.neurology.org//cgi/collection/guillainbarre_syndrome

Peripheral neuropathy

http://nn.neurology.org//cgi/collection/peripheral_neuropathy

Information about reproducing this article in parts (figures,tables) or in its entirety can be found online at:

http://nn.neurology.org/misc/about.xhtml\#permissions

Information about ordering reprints can be found online:

http://nn.neurology.org/misc/addir.xhtml\#reprintsus

Neurol Neuroimmunol Neuroinflamm is an official journal of the American Academy of Neurology.

Published since April 2014, it is an open-access, online-only, continuous publication journal. Copyright

Copyright $\odot 2020$ The Author(s). Published by Wolters Kluwer Health, Inc. on behalf of the American

Academy of Neurology.. All rights reserved. Online ISSN: 2332-7812.

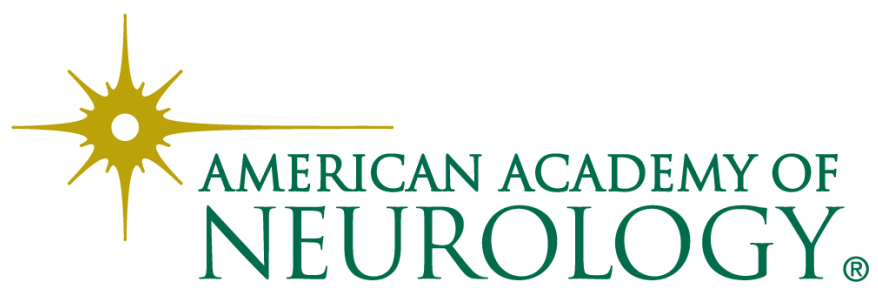

